\title{
Doença de Madelung: relato de caso e revisão da literatura*
}

Madelung's disease: a case report and literature review

\author{
Ligia Persici Rodrigues ${ }^{1}$, Ernesto Lima Araujo Melo²
}

\begin{abstract}
Resumo A doença de Madelung é caracterizada por massas simétricas de tecido adiposo no pescoço, ombros, braços e parte superior do tronco. A tomografia computadorizada demonstra achados de imagem característicos e é considerada o método de escolha para diagnóstico, estadiamento pré-operatório e acompanhamento pós-cirúrgico. Relatamos o caso de um homem com aspectos tomográficos típicos da doença de Madelung.

Unitermos: Doença de Madelung; Lipomatose simétrica múltipla benigna; Lipomas; Alcoolismo.
\end{abstract}

\begin{abstract}
Madelung's disease is characterized by the presence of symmetric masses of adipose tissue on the neck, shoulders, arms and the upper trunk. Computed tomography demonstrates characteristic imaging findings, and it is considered to be the method of choice for diagnosis, pre-operative staging, and postoperative follow-up. The authors report the case of a man with typical tomographic findings of Madelung's disease.

Keywords: Madelung's disease; Benign multiple symmetric lipomatosis; Lipomas; Alcoholism.

Rodrigues LP, Melo ELA. Doença de Madelung: relato de caso e revisão da literatura. Radiol Bras. 2012 Mar/Abr;45(2):129-131.
\end{abstract}

\section{INTRODUÇÃO}

Lipomatose simétrica múltipla, também conhecida como doença de Madelung ou síndrome de Launois-Bensaude, é uma entidade rara caracterizada pelo depósito subcutâneo indolor de tecido adiposo difusamente no pescoço, tronco superior, braços e pernas ${ }^{(\mathbf{1})}$.

Historicamente, a doença de Madelung tem sido vista mais em homens (proporção homem:mulher de 15:1) entre 30 e 60 anos de idade. Há aumento na prevalência da doença entre a população mediterrânea (incidência relatada na Itália de 1/25.000 homens), e existe relação entre essa doença e o consumo excessivo de álcool, particularmente o de vinho tinto ${ }^{(2)}$. Tal condição desenvolve-se durante um período de meses a anos. Os pacientes queixam-se de sua aparência, embora o aumento dos depósitos adiposos, em casos avançados, possa causar dispneia ou disfagia ${ }^{(3)}$. A doença de Madelung tem sido observada em associa-

* Trabalho realizado no Centro de Imagens do Hospital Geral Dr. César Cals (HGCC) - SESA, Fortaleza, CE, Brasil.

1. Médica Residente em Radiologia do Instituto Dr. José Frota, Fortaleza, CE, Brasil.

2. Doutor, Médico Radiologista do Hospital Geral Dr. César Cals (HGCC) - SESA, Fortaleza, CE, Brasil.

Endereço para correspondência: Dra. Ligia Persici Rodrigues. Rua Pedro Rufino, 100, 802C, Varjota. Fortaleza, CE, Brasil, 60175-100. E-mail: ligia_persici@hotmail.com

Recebido para publicação em 19/6/2011. Aceito, após revisão, em 29/10/2011. ção com diabetes, gota, alcoolismo e outras doenças sistêmicas $^{(4)}$.

Relatamos o caso de um homem de 50 anos de idade apresentando massas indolores cervicofaciais e nas regiões superiores do tronco, que aumentaram gradualmente de volume ao longo de três a quatro anos (Figura 1).

\section{RELATO DO CASO}

O histórico médico do paciente era marcado por hipertensão arterial sistêmica, controlada efetivamente com medicação. Negava aumento do aporte calórico e seu consumo alcoólico fora maior que 1,0 litro de bebida destilada por dia, durante 28 anos. No exame físico, havia zonas de au- mento de volume na face, pescoço e tronco superior, sem sinais de edema. Subsequentemente, o paciente submeteu-se a tomografia computadorizada (TC) do crânio e pescoço, com a hipótese clínica de "lipomas".

Os achados de TC mostraram distribuição simétrica e difusa de material adiposo homogêneo não encapsulado no subcutâneo da região cervicofacial e nas porções superiores do tronco, sem evidências de realce pós-contraste. Os planos musculares profundos demonstraram formas, contornos e coeficientes de atenuação preservados, sendo levemente rechaçados pelo material adiposo e sem, no entanto, exibir sinais de infiltração direita. Não havia linfonodomegalias, massas com coeficientes

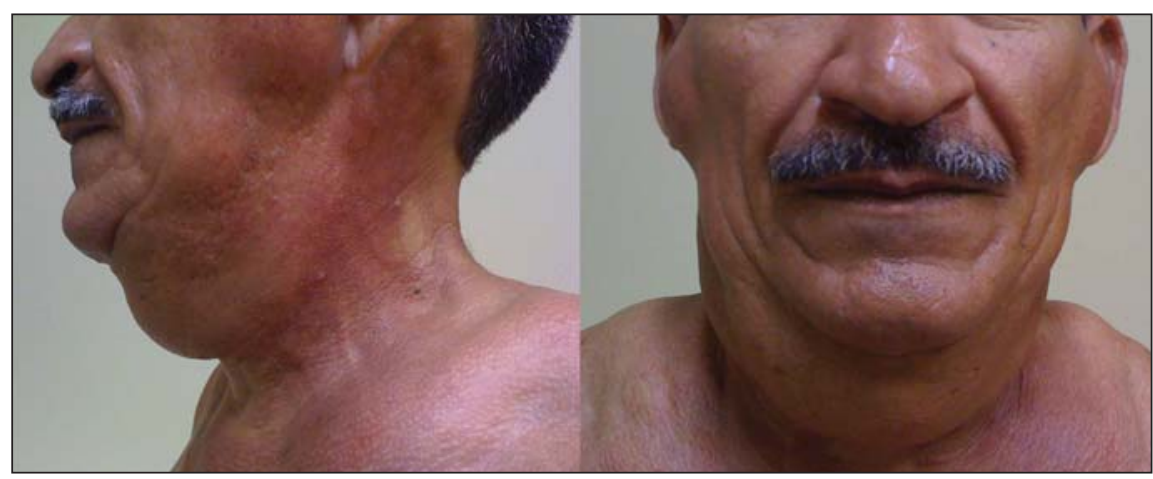

Figura 1. Imagens lateral e frontal do paciente mostrando aumento simétrico dos tecidos moles decorrente do depósito de material adiposo, mais evidente nas regiões pré-auriculares, submandibulares e supraclaviculares. 


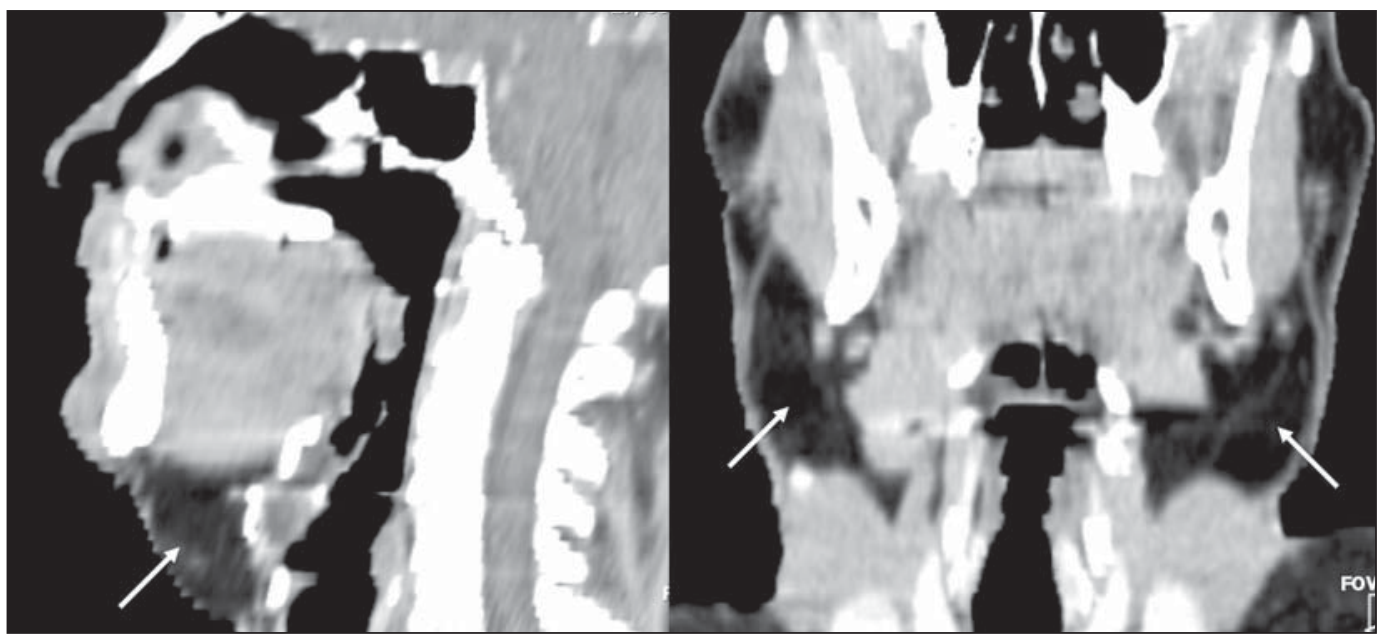

Figura 2. Reconstruções sagital e coronal mostrando deposição profunda e difusa de tecido adiposo (setas). de atenuação de partes moles, francas malformações linfáticas ou dilatações vasculares (Figura 2).

\section{DISCUSSÃO}

Madelung foi o primeiro a extensivamente descrever essa entidade numa série de pacientes em 1888, e a doença é referida como doença de Madelung. Launois e Bensaude relataram 65 casos em 1889, expandindo a descrição dessa condição, que tem sido referida também como síndrome de Launois-Bensaude. Atualmente, mais de 270 casos foram descritos ${ }^{(\mathbf{2 , 5})}$.

A lipomatose simétrica múltipla é classificada em dois tipos. No tipo I, há massas lipomatosas nas regiões parotídeas, no pescoço, na região supraescapular e no deltoide. Neste grupo, em alguns pacientes pode haver acometimento profundo, causando dispneia e disfagia. No tipo II, a lipomatose é difusa, com a aparência clínica de obesidade simples, não apresentando os sintomas de envolvimento profundo que podem acontecer no tipo $\mathrm{I}^{(\mathbf{6})}$. As massas normalmente não respeitam planos de clivagem, penetrando em todos os níveis, dificultando sua exérese cirúrgica ${ }^{(4)}$.

Vários tipos de lipomatoses, normalmente diferenciados por localização anatômica e variadas manifestações clínicas, têm sido descritos, como a lipomatose simétrica múltipla, lipomatose congênita infiltrativa da face, lipomatose encefalocraniocutânea, lipomatose da cintura escapular, adipose dolorosa, lipomatose pélvica e lipomatose mediastinal ${ }^{(5)}$. O diagnóstico clínico é feito pelo reconhecimento da aparência única do(a) paciente, mas, em casos atípicos, pode haver confusão com doenças linfoproliferativas e outras condições ${ }^{(4)}$.

A etiologia da doença de Madelung é desconhecida. A ingestão de álcool de mais de $80 \mathrm{~g}$ por dia durante pelo menos 10 anos, encontrada em até $90 \%$ dos casos descritos na literatura, também estava presente neste relato de caso. Polineuropatia, descrita como uma disfunção sensorial, motora e autonômica, e detectada em cerca de $85 \%$ dos pacientes com doença de Madelung, geralmente se desenvolve vários anos após o aparecimento dos lipomas e não estava presente no paciente em questão ${ }^{(7)}$.

A radiografia de tórax pode mostrar alargamento mediastinal. Na TC observase tecido lipomatoso não-encapsulado e homogêneo, podendo haver deposição pro- funda (submuscular) de tecido adiposo (Figura 3). Esta última apresentação ocorre mais frequentemente em volta da traqueia e/ou profundamente ao músculo trapézio. Em alguns casos, identificam-se calcificações no interior dos lipomas ${ }^{(7)}$.

A TC é considerada, atualmente, o método de escolha para o diagnóstico, estadiamento pré-operatório e acompanhamento pós-cirúrgico dos doentes com lipomatose simétrica múltipla. A avaliação das imagens diretamente no monitor do tomógrafo, em estações de trabalho ou em computadores dotados de programas visualizadores, permite a modificação dinâmica de parâmetros de janelas e níveis, além da mensuração dos coeficientes de atenuação em áreas de interesse, o que pode melhorar a identificação e caracterização do material adiposo. Apesar de não terem sido comentadas mesmo

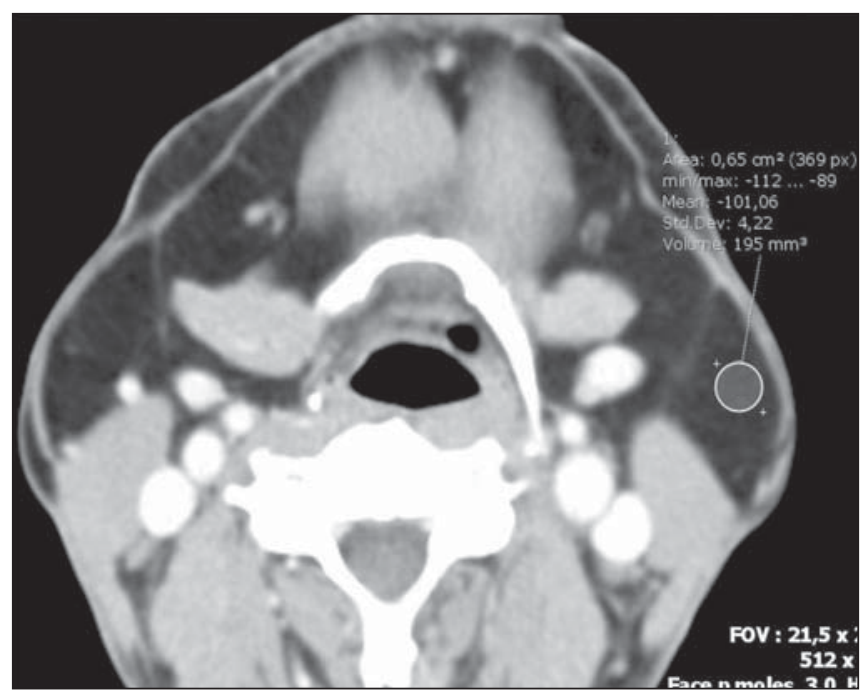

Figura 3. Imagem axia pós-contraste mostrando depósito de tecido adiposo difuso envolvendo estruturas profundas. 
em revisões recentes ${ }^{(8)}$, algumas das vantagens da TC incluem as reformatações multiplanares, pois, ainda que prescindíveis para o diagnóstico, podem refinar a apresentação dos achados e facilitar a apreciação da distribuição da lipomatose para radiologistas e não radiologistas (Figura 2).

A avaliação por TC permite afastar importantes diagnósticos diferenciais, tais como linfoma e comprometimento metastático linfonodal. A TC também possibilita o estadiamento tumoral nos casos em que a lipomatose simétrica múltipla esteja associada a doença maligna, especialmente o carcinoma de células escamosas das vias aéreas superiores, decorrente de etilismo e tabagismo muitas vezes associados ${ }^{(\mathbf{9})}$.

A aparência ultrassonográfica das lipomatoses tem aspecto típico. Porém, apesar de a ultrassonografia ser amplamente uti- lizada na investigação de massas cervicais, as medidas de densidade realizadas por TC têm maior fidelidade diagnóstica ${ }^{(6)}$.

Os achados na ressonância magnética (RM) quase sempre se sobrepõem aos da TC, ressaltando-se, porém, que a RM é mais onerosa e geralmente menos acessível ${ }^{(9)}$.

Apesar de ter incidência relativamente baixa, os radiologistas devem conhecer os achados de imagem típicos da doença de Madelung, para estabelecer prontamente o diagnóstico correto, excluir outras condições que se sobrepõem, e fornecer informações adequadas para um manejo médico pertinente.

\section{REFERÊNCIAS}

1. Feliciani C, Amerio P. Madelung's disease: inherited from an ancient Mediterranean population? N Engl J Med. 1999;340:1481.

2. Landis MS, Etemad-Rezai R, Shetty K, et al. Case
143: Madelung disease. Radiology. 2009;250: 951-4.

3. Josephson GD, Sclafani AP, Stern J. Benign symmetric lipomatosis (Madelung's disease). Otolaryngol Head Neck Surg. 1996;115:170-1.

4. Ramos GHA, Trevizan GL, Pepe ES. Doença de Madelung. Rev Bras Otorrinolaringol. 2002;68: 587-90.

5. Murphey MD, Carroll JF, Flemming DJ, et al. From the archives of the AFIP: benign musculoskeletal lipomatous lesions. Radiographics. 2004;24:143366.

6. Vieira MV, Grazziotin RU, Abreu M, et al. Lipomatose simétrica múltipla (doença de Madelung): relato de um caso. Radiol Bras. 2001;34:119-21.

7. Bulum T, Duvnjak L, Car N, et al. Madelung's disease: case report and review of the literature. Diabetologia Croatica. 2007;36:25-30.

8. Vidal MGC, Haygert CJP, Zagoury AR, et al. Doença de Madelung: relato de caso e revisão da literatura. Radiol Bras. 2010;43:275-6.

9. Souza RP, Paes Junior AJO, Rapoport A. Doença de Madelung (lipomatose cervical benigna simétrica): relato de dois casos. Radiol Bras. 2003;36: 121-2. 